\title{
Three-dimensional visualisation of fatigue cracks in metals using high resolution synchrotron X-ray micro-tomography
}

\section{J. Y. Buffiere*, E. Ferrie, H. Proudhon and W. Ludwig}

The present paper reviews recent developments in high resolution synchrotron X-ray microtomography for the study of fatigue cracks in metals. The possibilities and limitations of the tomography technique are described. A fatigue machine used for in situ cycling of samples is presented and three-dimensional images of the growth of fatigue cracks initiated in various Al alloys on natural or artificial defects are shown. Examples of quantitative use of such 3D images are given.

Keywords: 3D fatigue crack, in situ, Synchrotron X-ray tomography

\section{Introduction}

Although a lot of experimental and theoretical efforts have been spent in the characterisation and modelling of small fatigue crack propagation (see for example Newman ${ }^{1}$ for a recent review), the growth rate predictions for this kind of defects are far from being completely satisfactory for the moment.

At least three different classes of small fatigue cracks can be distinguished, ${ }^{2}$ in what follows, $a$ stands for the crack size and $r_{\mathrm{p}}$ for the plastic zone size:

(i) microstructurally small cracks for which $a \approx \lambda$ where $\lambda$ represents the size of a characteristic feature of the alloy microstructure (grain, reinforcing particles, ...)

(ii) mechanically small cracks for which $a<r_{\mathrm{p}}$ (in that particular case $r_{\mathrm{p}}$ is either the crack plastic zone size itself or that of a notch)

(iii) physically small cracks for which $a \gg r_{\mathrm{p}}$ and $a$ is typically $<500 \mu \mathrm{m}$; in that case crack closure is very limited inducing high values of cyclic growth rate.

Finally, cracks with a size larger than $500 \mu \mathrm{m}-1 \mathrm{~mm}$ and verifying $a \gg r_{\mathrm{p}}$ are called long cracks; their propagation can be described in the frame of the linear elastic fracture mechanics via the use of the Paris law. ${ }^{1}$

One very basic problem caused by small fatigue cracks is that their complex three-dimensional (3D) shapes affect their propagation behaviour. Therefore, the propagation at the surface of a fatigue sample is not necessarily representative of the bulk behaviour, on the contrary of long cracks, typically more than several millimetres in length. Although this problem has been recognised at least 20 years ago it has hardly been taken into consideration in practice. Design against fatigue in

GEMPPM INSA Lyon 20 Av. A. Einstein 69621 Villeurbanne Cedex, France

*Corresponding author, email jean-yves.buffiere@insa-lyon.fr industrial components sometimes includes technical specifications to account for small fatigue crack growth, mostly on the basis of empirical models. ${ }^{3}$ The resolution of experimental techniques such as beach marking ${ }^{4}$ or heat tinting ${ }^{5}$ conventionally used to visualise the shape of long cracks is not sufficient to visualise the $3 \mathrm{D}$ shape of small cracks. Therefore, experimental characterisation of small fatigue cracks $3 \mathrm{D}$ growth has, up to now, remained very limited.

For such a characterisation, X-ray micro-tomography is a very attractive technique which enables the visualisation of internal features in opaque samples. Being a non-destructive technique, it also enables, in principle, in situ visualisation of damage during loading and provides therefore the chronology of damage initiation and growth. In the last 10 years, significant progress has been made in terms of resolution with the availability of both new third generation synchrotron Xray sources and new detectors. ${ }^{6}$ Spatial resolution close to that of an optical microscope can now be achieved in 3D which opens (or reopens) wide areas of research.

The present paper is a review of experiments carried out in the last eight years at the European Radiation Synchrotron Facility (ESRF). It aims at illustrating the potential of high resolution synchrotron X-ray microtomography for the study of fatigue damage development in metallic materials under cyclic loading; the limitations of the technique in terms of spatial resolution versus sample size are underlined and experimental set-ups/methods are described. Examples of 3D reconstructed images are given with an emphasis on how these images can be used to validate/test existing models in the field of fatigue.

\section{Experimental}

\section{Tomography set-up at ESRF}

All the experiments described in the present paper have been performed at the ESRF in Grenoble (France) on beam line ID19. This beam line is one of the five which 
offer, for the moment, the possibility of performing 3D imaging at the ESRF; other beam lines offer different characteristics such as high energy for beam lines ID15 and ID11, fluorescence imaging for beam line ID21, etc.

It is not the aim of the present paper to describe in detail the tomography experimental set-up of ID19 which can be found elsewhere, ${ }^{7}$ however, in what follows, some important features are briefly recalled which will be used further for discussing the limits of the technique.

The permanent micro-tomographic set-up has been designed to perform experiments giving $3 \mathrm{D}$ images with a voxel* size ranging from $0.3 \mu \mathrm{m}$ to several tens of micrometres according to the optics used. Obtaining a tomographic image consists first in recording a series of $\mathrm{N}$ radiographs of a sample which is rotated around one axis (generally set vertical). These $\mathrm{N}$ radiographs are then used by a reconstruction algorithm to obtain a $3 \mathrm{D}$ numerical image of the sample which is, in its classical form, a 3D map of the attenuation coefficient in the sample. $^{8}$

The two-dimensional (2D) radiographs which compose the tomographic scan of the studied samples are recorded on a detector developed at the ESRF. ${ }^{9}$ It consists in a fast readout $(60 \mathrm{~ms})$, low noise (Frelon) charge coupled device (CCD) camera with a square array of $1024 \times 1024$ or $2048 \times 2048$ pixels. This detector is coupled with a fluorescent screen via optical lenses. The white beam coming out of the synchrotron ring is rendered monochromatic by a multilayer monochromator. The energy of the beam can be tuned from 10 to $\sim 60 \mathrm{keV}$. The beam coming out of the monochromator is a parallel beam so that no geometric enlargement is possible; instead, the voxel size is a result of the optics used.

Another important feature of beam line ID19 is the unusually long source to specimen distance (145 m) which allows one to perform phase contrast imaging in a very simple way by increasing the sample to detector distance. Finally, the space available in front of the CCD detector enables to install reasonably bulky experimental devices such as cold cells, furnaces or mechanical testing devices for applying various experimental conditions to the samples in situ during tomographic imaging. For the work presented in the present paper, one special fatigue machine has been designed in order to load mechanically the samples. Its main features are described in the next section.

\section{In situ fatigue machine}

The in situ fatigue machine has been designed at INSA and fulfils three requirements:

(i) its size must be as small as possible in order to reduce as much as possible the sample to detector distance

(ii) the level of vibration induced by the loading/ unloading cycles and the weight of the machine must be kept as small as possible in order to avoid damaging the precision mechanics sample stage used to rotate the sample. In addition the weight distribution around the stress axis should

*A voxel is the smallest elementary numerical element composing 3D images. It is therefore the $3 \mathrm{D}$ equivalent of the pixel in classical two dimensional images.

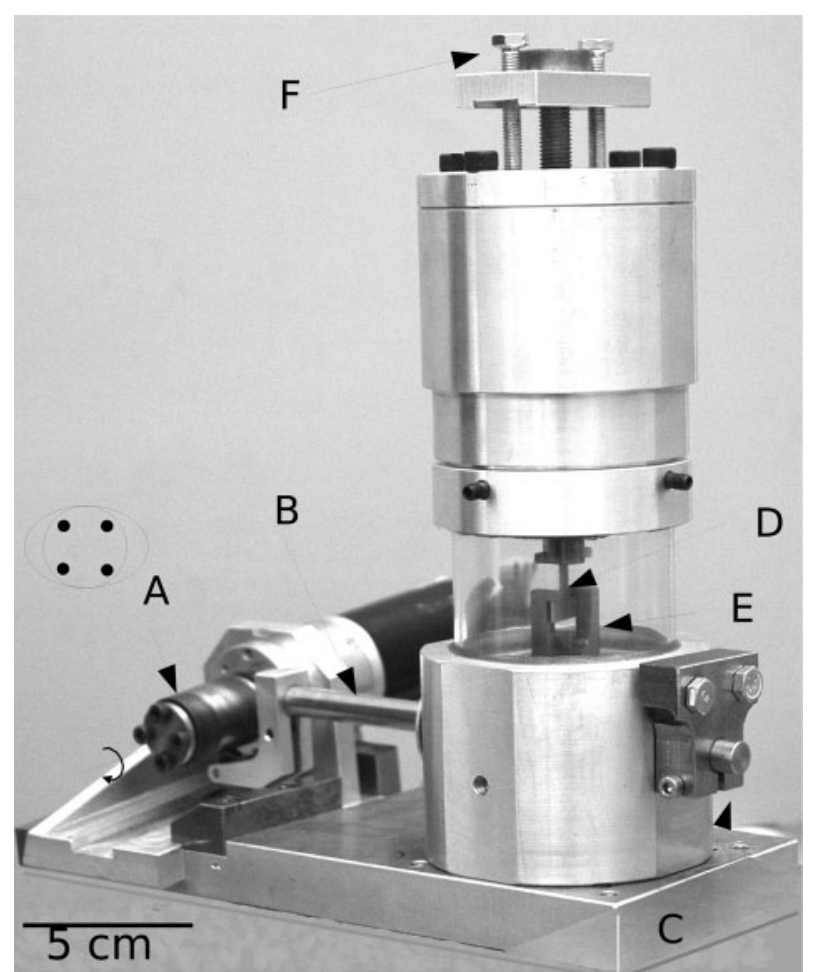

1 Picture of fatigue machine designed for in situ cycling of samples during micro-tomography experiments: rotating cam $A$ actuates lever $B$ which is fixed at one end $C$; elliptical cross-section of cam induces loading/ unloading of sample $D$ which is attached to actuating lever by partially visible grip $E$; value of minimum cyclic load is fixed by using two screws $F$ attached to upper grip; schematic drawing in black on left shows section of cam as seen along rotation, indicated by broken line; polymer tube (PMMA) is used to transmit load from bottom to top of machine; cycling frequency of $50 \mathrm{~Hz}$ can be obtained while maintaining vibration level low so that machine can be installed directly on sample stage of micro-tomography set-up

be kept as symmetrical as possible to reduce parasitic movement (wobble) when the sample/ machine is moved momentarily out of the beam for recording reference images

(iii) a cyclic frequency larger than $35 \mathrm{~Hz}$ is desirable in order to perform a number of fatigue cycles of the order of $10^{6}$ during one shift $(8 \mathrm{~h})$ of beamtime.

To fulfil the last requirement, mechanical loading of the sample has been applied by a rotating cam. This cam which has a complex elliptical/conical shape is visible in Fig. 1 (detail A) which shows a picture of the machine. The cam rotation movement is transformed into a translation movement via an actuating lever (detail B) which is fixed on one side of the machine (detail C) and on which the sample (detail D) is attached via a special grip (detail E). The value of the minimum cyclic load is adjusted manually using two screws attached to the upper grip (detail F). The elliptical cross-section of the cam, section perpendicular to the cam rotation axis (see figure 1), induces loading/unloading cycles in the sample while the conical section, section containing the cam rotation axis, is used to change the cyclic stress amplitude by moving the cam perpendicular to the actuating lever. The load is transmitted from the top to 
the bottom of the machine via a thin perspex tube* ( $1 \mathrm{~mm}$ thick, $60 \mathrm{~mm}$ in diameter) which allows a $180^{\circ}$ rotation without hiding the sample and which results in a constant but negligible attenuation of the X-ray beam. The machine weights $6 \mathrm{~kg}$ and can therefore be safely installed on the sample stage.

\section{Limitations of micro-tomography for characterisation of fatigue cracks}

\section{Sample size}

Cracks can be imaged in tomography because they introduce a local variation of the attenuation coefficient within a material. However, the distance between the two crack faces is small: at the back of the crack tip it is typically of the order of $1 \mu \mathrm{m}$ if the crack has millimetric dimensions while at the crack tip the crack opening is at the nanometric scale. The authors' past experience on the visualisation of fatigue cracks by tomography showed that a $6 \mu \mathrm{m}$ voxel size was not sufficient to image microstructurally small fatigue cracks, typical size less than $100 \mu \mathrm{m}$, initiated on pores in a cast $\mathrm{Al}$ alloy ${ }^{10}$ while a voxel size close to $1 \mu \mathrm{m}$ did allow the detection of similar cracks in $\mathrm{Fe}^{11} \mathrm{Al}^{7}$ or $\mathrm{Ti}^{12}$ based materials. With the parallel beam used on ID19, the maximum size of a sample whose projections entirely fit on the detector is directly given by the product of the voxel size in the reconstructed image $v_{\mathrm{s}}$ by the number of elements $n$ of the CCD. For a voxel size of $0.7 \mu \mathrm{m}$ and the $2048 \times 2048$ Frelon camera, this gives a maximum sample size of $1.4 \mathrm{~mm}$ which corresponds to a square cross-section of $1 \times 1 \mathrm{~mm}^{2}$. In a metallic material with a typical grain size of the order of $100 \mu \mathrm{m}$ this implies that a stable fatigue crack nucleated and propagated in situ can at best cross between 10 and 20 grains and is therefore a microstructurally small crack. ${ }^{13}$ Performing a tomographic scan on a sample whose size is larger than $v_{\mathrm{s}} n$ (a method called local tomography) has been tried by various researchers and seems to give reasonably good results as long as the part of the sample which falls outside of the field of view is not too heterogeneous and does not alter too much the recorded projections. Whether this technique could be used to image a fatigue crack, which is a rather strong inhomogeneity in the sample, is not known for the moment; experiments aiming at clarifying this point are planned.

However, even if local tomography proves to be successful for imaging crack tips within large samples, one intrinsic limitation of the tomography technique remains the efficiency of the imaging detector at high energy. Indeed the high spatial resolution achieved in the reconstructed images depends on the thickness of the fluorescent screen which has to be kept as thin as possible to avoid lateral spreading of the signal when a photon impinging on the screen is turned into visible light. Thin fluorescent screens tend to be 'transparent' to high energy photons. In practice, assuming that the efficiency of the fluorescent screen used for imaging with a $0.7 \mu \mathrm{m}$ voxel size falls off quickly above an energy of $30 \mathrm{keV}$, a simple attenuation calculation shows that the maximum sample size which can be crossed while keeping a 10\% transmission of the incident beam is $8 \mathrm{~mm}$ for $\mathrm{Al}$ and only $1 \mathrm{~mm}$ for iron. This explains why at the moment fatigue cracks

* If metallic poles were used as in classical tension/compression rigs, they would hide the sample from the beam during the 180 degree rotation.

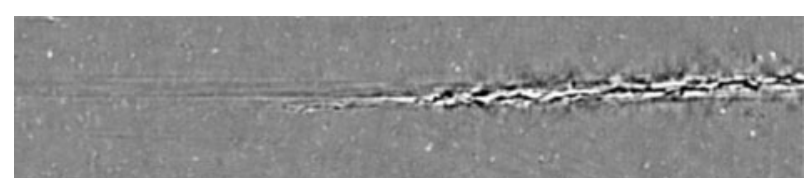

$50 \mu \mathrm{m}$

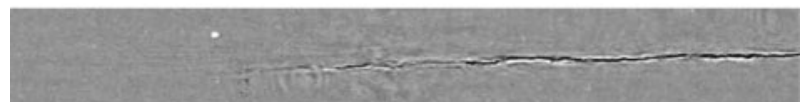

2 Reconstructed images of fatigue crack in Al-Li alloy with fine grain size $(\sim 1 \mu \mathrm{m})$ using two different specimen detector distances: top - sample/detector distance is $35 \mathrm{~mm}$, bottom - sample/detector distance is $5 \mathrm{~mm}$; smaller specimen/detector distance results in lower level of phase contrast in reconstructed images which are therefore easier to process

with a size of several millimetres can only be imaged in 3D at high resolution in light alloys.

\section{Phase contrast imaging: advantages and drawbacks}

It was mentioned before that phase contrast imaging can easily be used on ID19. This method of imaging has been shown to be very efficient for improving damage detection. ${ }^{14}$ However, if phase contrast does help to underline defects such as cracks and therefore considerably facilitates a visual online detection during an in situ experiment, the presence of diffraction fringes on the crack edges and at the crack tips can complicate a quantitative analysis of the crack shape. An example is given in Fig. 2 which shows two reconstructed images of a fatigue crack in a very fine grain $(\sim 1 \mu \mathrm{m}) 5091 \mathrm{Al}-\mathrm{Li}$ alloy. The image at the top (Fig. 2a) has been obtained while keeping the sample under load in a tensile testing device fitted with a polymer tube similar to the one shown in Fig. 1. Owing to the presence of the tube, the detector to specimen distance could not be $<35 \mathrm{~mm}$. In the image at the bottom (Fig. 2b), the polymer tube has been removed, the sample is not under load but the specimen/ camera distance has been reduced to $5 \mathrm{~mm}$. When doing so, the amount of phase contrast in the reconstructed image is considerably reduced, the crack edges look sharper and are easier to define and a quantitative analysis like automatic threshold of the crack shape is therefore easier to perform. In principle it is possible to suppress the presence of phase contrast fringes on reconstructed images by recording several scans (two or three) of the same sample at several specimen/detector distances. A dedicated reconstruction algorithm is then used to reconstruct the phase shifts induced by internal features in the material (a process called holotomography ${ }^{15}$ ). But this solution is not well suited for in situ experiments where it is typical to record between 10 and 20 scans to monitor the growth of a fatigue crack; the amount of data generated by holotomography would therefore be too large to be used in practice.

It must be pointed out finally that the very flat shape of the crack shown in Fig. 2, which is a result of the fine microstructure of the alloy, emphasises the drawbacks of the phase contrast and represents probably one of the worst case that can be encountered when imaging cracks by tomography. Indeed, in conventional alloys, as the $3 \mathrm{D}$ crack shapes are more tortuous (larger grain size), the crack edges 


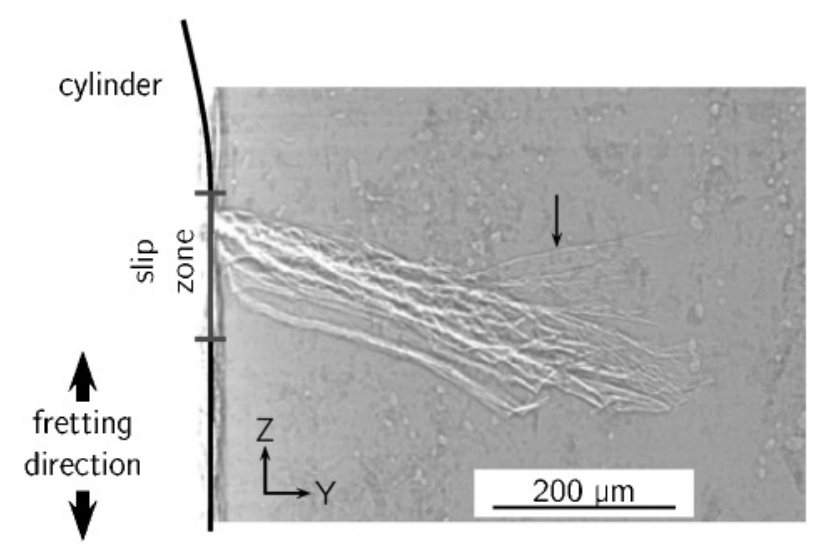

3 Two-dimensional radiograph of fretting crack in 2024 Al alloy: on right side of micrograph line gives shape of cylinder used for performing fretting experiment; many branches visible on image corresponding to different planes of propagation in grains present within thickness of sample

tend to be better defined (less reconstruction artefacts) even when the sample is imaged in a polymer tube. ${ }^{16}$

\section{Examples of results}

\section{Crack initiation from natural defects}

Fatigue cracks in their large majority initiate from defects which create elastic/plastic inhomogeneities in the material. Post-mortem fractographic observations of samples broken during uniaxial fatigue reveal that the initiating defects are generally at or close to the sample surface*. This is because in that case the stress concentration effect increases owing to the inhomogeneities. $^{17,18}$ What is difficult to assess from post-mortem analysis, however, is the chronology of fatigue and, more precisely, the chronology of the first propagation stages which transforms the defect into a crack whose growth rate can be described, for example, via a Paris law. A few experimental evidences can be found in the literature which show that this transient process is threedimensional, i.e. that the development of a microstrurally small crack is hard to describe from simple surface observations. ${ }^{19-21}$ However, no systematic study has been undertaken so far in this field, mainly because the experimental characterisation of this problem is painstaking when classical methods (serial polishing, marker technique...) are used.

Compared with those methods micro-tomography appears to be a powerful technique. Some results have been obtained on ID19 in the last five years, for example on cast $\mathrm{Al}$ alloys, ${ }^{7,10,16}$ or cast iron. ${ }^{11,22}$ They show that during uniaxial fatigue the probability of initiating a crack from a porositylike defect is strongly correlated with the distance of the defect to the surface and that initiation from defects located at a distance larger than the defect size is quite rare. ${ }^{22}$ Another interesting point shown by $3 \mathrm{D}$ images is that although nearly all defects intersecting the surface do initiate a crack, most of them will remain

*In more complex loading cases like contact stresses the maximum stress level appears underneath the sample surface and therefore the most critical defects are located below the surface.

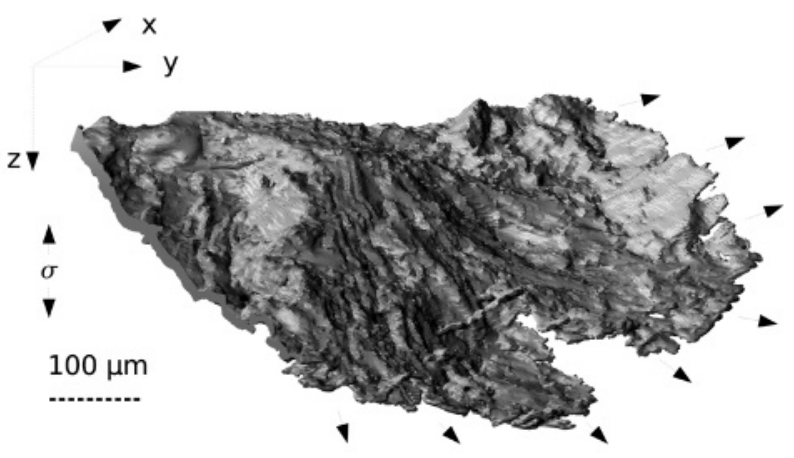

4 Three-dimensional rendition of small fatigue crack in cast Al alloy: grain size is $\sim 300 \mu \mathrm{m}$; presence of grain boundaries strongly influences crack path which is not flat; direction of stress applied during fatigue cycle is along $\mathbf{Z}$ axis; arrows indicate local crack growth direction along crack front

non-propagating: ${ }^{10}$ the driving force for microstructurally small crack propagation in the vicinity of the defect being probably high enough for initiation but too low for propagation. ${ }^{10,11,19}$ Finally, detailed observations of the development of a microstructurally fatigue crack around a porosity in a cast $\mathrm{Al}$ alloy have shown that a substantial part of the fatigue life can be spent in transforming the initiating pore into a crack, a process which is non-visible from the surface. During this transient stage, grain boundaries have also been observed to play a significant role as obstacles which slow down crack propagation. ${ }^{16}$

\section{Crack initiation from artificial defects}

Although the above mentioned in situ studies of naturally occurring fatigue cracks provide unique experimental data which are very important to understand the early stage of fatigue crack propagation, their major drawback is that at low stress levels a very large number of fatigue cycles are generally necessary to initiate a crack. Consequently, a long time is spent scrutinising 2D radiographs of the sample gage length before the first 3D image can be recorded. A solution to this problem is to create a small defect which can then be monitored by tomography. An example of such defect in a $2024 \mathrm{Al}$ alloy is shown in Fig. 3. In this case, a fatigue crack was created in the sample before the tomography experiment by fretting fatigue. The fretting fatigue experiment was performed to simulate crack initiation at the contact between a rivet and a fuselage plate of a plane. The aim of the subsequent uniaxial loading was to simulate the propagation of the microstructurally small crack in the far tensile stress field of the plane fuselage (see Ref. 23 for details).

The possibility of creating tiny artificial notchlike defects by focussed ion beam (FIB) machining has also been investigated. A shallow notch $(100 \mu \mathrm{m}$ wide, $25 \mu \mathrm{m}$ deep and $2 \mu \mathrm{m}$ thick) has been machined in a fine grain 5091 Al-Li alloy. After in situ cycling this notch initiated a crack whose development will be illustrated in the next section. Besides saving time for monitoring crack initiation, such model defects are also very interesting for studying the intermediate crack shapes corresponding to the transformation of a notch of a given shape in a crack. It must be pointed out finally that in most cases FIB machining uses heavy gallium ions. In the case of 


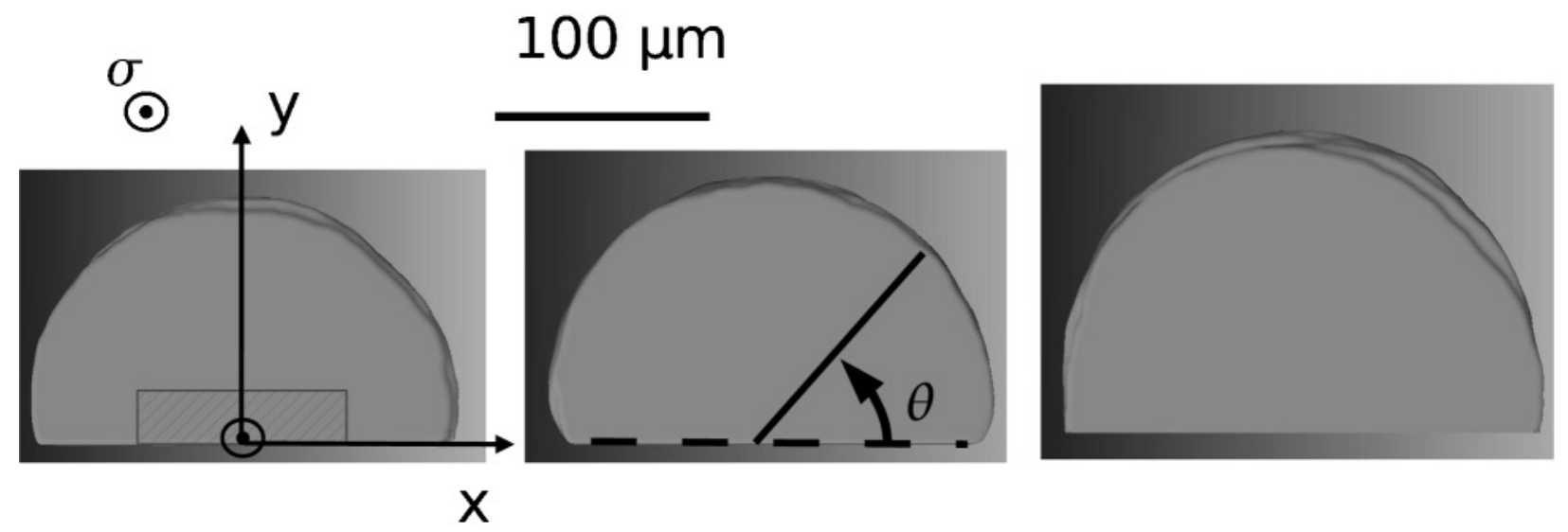

5 Three-dimensional renditions of crack, as seen along stress direction, growing in mode I inside millimetric sample of fine grain $(1 \mu \mathrm{m})$ Al-Li alloy: because of small grain size, crack looks extremely flat with very regular crack front; position along crack front is defined via angle; surface of sample corresponds to broken line visible on middle image, $=0^{\circ}$; crack is initiated from artificial notch machined by FIB and whose shape is shown by hatched rectangle on left image

the fine grain alloy studied here, no embrittlement effect induced by gallium was noticed but that may not necessarily be the case for other $\mathrm{Al}$ alloys. ${ }^{24}$

\section{Propagation of microstructurally small cracks}

Figure 4 shows a typically reconstructed image of the surface of a microstructurally small fatigue crack (the surrounding metal has been set to transparent) as observed after 325000 cycles of stable propagation in a cast $\mathrm{Al}$ alloy. For this material the average grain size was $\sim 300 \mu \mathrm{m}$, i.e. of the order of the crack size observed in this figure. The non-planar shape of the crack plane seen in this figure is typical of the interaction of microstructurally small cracks with grains. By using a gallium infiltration technique ${ }^{7}$ it is possible to make the grain boundaries surrounding a cracks visible on a $3 \mathrm{D}$ reconstructed image, and therefore to correlate irregularities of the crack front to those grain boundaries. It was possible to show, for example, that protruding or retarded parts of the crack front were correlated with the presence of grain boundaries. ${ }^{16,25}$ The ability of a microstructurally small crack to propagate faster/slower in some grains is probably linked to the ease/difficulty of activating small scale plasticity in the adjacent grains as suggested by Newman. ${ }^{1}$ The knowledge of the local crystallographic orientation would be necessary to check this assumption. In principle this orientation could be obtained by three-dimensional X-ray diffraction (3DXRD), a non-destructive characterisation technique also developed at ESRF. ${ }^{26}$ This technique gives access to the 3D shape and orientation of grains in the bulk of undeformed polycrystals provided their size is not to small $(>50 \mu \mathrm{m})$ and their mosaicity not too large $\left(<1^{\circ}\right)$. Such experiments are currently underway.

Variations in the activation of local plasticity along the crack front depend not only on the local crystallography but also on the local stress level which in turn is a function of the local crack and sample shape and dimensions via the stress intensity factor $K$. The evolution of $K$ along the irregular front of 3D microstructurally small fatigue cracks observed by micro-tomography has been calculated using extended 3D finite elements (XFE). ${ }^{27}$ The FE mesh is directly constructed from the $3 \mathrm{D}$ reconstructed image. The calculated $K$ values are the results of an elastic calculation. This is a rather strong assumption given the small size of the considered cracks. Nevertheless, such an elastic calculation shows clearly that protruding parts of the crack front induce a reduction of the $K$ level while retarded parts induce an increase in $K$. This result had already been obtained by approximate analytical calculations on simple planar cracks. ${ }^{28}$ Therefore, any propagation anisotropy induced along the crack front by the local crystallography should tend to equilibrate itself along the crack front and to eventually vanish when the crack is large enough. For the reasons evoked before (limited field of view) it has not been possible yet to image the transition from the microstructurally small crack regime to the long crack regime.

Instead, a simulation of a 'long crack' experiment has been carried out on a $5091 \mathrm{Al}-\mathrm{Li}$ alloy ${ }^{27}$ with an ultra fine grain size $(\sim 1 \mu \mathrm{m})$. The advantage of such a fine microstructure is twofold. First, the number of grains encountered by the front of a crack with a typical size of a few hundred micrometres is comparable with that encountered by a long (several millimetres) crack in a material with a $50 \mu \mathrm{m}$ grain size. Second, the crack shape in this kind of material is known to be extremely $\mathrm{flat}^{29}$ and therefore XFE modelling of the crack is easier to perform.

\section{Propagation of 'long' fatigue cracks}

Figure 5 shows three stages of propagation of such a crack which has been initiated from the FIB notch mentioned in the previous section. A detailed description of this experiment can be found elsewhere. ${ }^{27}$ The local propagation rate along the crack front as defined by the angle in Fig. 5 was measured. For $\theta=90^{\circ}$ the propagation rate was found to be larger for equivalent crack sizes than for $\theta=0^{\circ}$ (surface of the sample). It is worth pointing out that when plotting the crack propagation rate for $\theta=0^{\circ}$ on a $\mathrm{d} a / \mathrm{d} N=\mathrm{f}(K)$ curve, the experimental values obtained for the submillimetric crack superimpose on those obtained for long cracks in large compact tension samples for the same material. ${ }^{27}$ This confirms that the in situ experiment in a $1 \mathrm{~mm}$ sample can simulate a long crack experiment, provided the grain size is small enough compared with 
the crack size. By taking into account the evolution of the crack closure stress along the crack front, it was possible to superimpose the $\mathrm{d} a / \mathrm{d} N$ values for $\theta=0^{\circ}$ and $\theta=90^{\circ}$ assuming that the closure level of the crack varied linearly between the surface (maximum closure level) and the bulk (no closure).

Finally, the nearly semicircular shape of the crack observed in Fig. 5 is not expected/observed for long edge cracks which tend to grow with elliptical shapes (constant $K$ ). ${ }^{30}$ It is the result of the higher closure level at the surface which slows down crack growth compared with crack growth in the bulk. ${ }^{28}$

\section{Conclusions and perspectives}

The aim of the present paper was to review recent experiments of X-ray micro-tomography imaging to study in situ the growth of fatigue cracks. Two limitations of the technique have been described: the small size of the studied samples and the presence, on reconstructed images, of strong reconstruction artefacts arising from phase contrast.

An in situ loading device that can be mounted directly on the tomography set-up of beam line ID19 at ESRF has been described. It enables high frequency $(50 \mathrm{~Hz})$ cycling and the interrupted in situ observation of microstructurally small fatigue crack growth - with a size typically ranging from 10 to $500 \mu \mathrm{m}$ - initiated on natural or artificial defects.

In $\mathrm{Al}$ alloys, the analysis of reconstructed $3 \mathrm{D}$ images has shown that the presence of grain boundaries strongly influences the crack front shape which becomes very irregular. The post-mortem visualisation of the $3 \mathrm{D}$ grain shape, via a $\mathrm{Ga}$ infiltration technique, clearly shows this interaction. However, further modelling of the behaviour of such small cracks would require the knowledge of the local crystallographic orientations.

Finally, 'long cracks' with a very regular shape have been obtained in an Al-Li aluminium alloy with a small grain size $(1 \mu \mathrm{m})$. The propagation of such cracks has been modelled using XFE methods and local variations of the stress intensity factors along the crack front have been obtained and correlated with the experimental local crack growth rate.

In the future, metallic materials with an intermediate grain size $(20-50 \mu \mathrm{m})$ will be studied in order to visualise the transition between small and long crack growth. This kind of observation should provide valuable experimental input to crack propagation models which aim at describing the growth of fatigue cracks from the early stages of crack initiation to the final fracture.

\section{Acknowledgements}

The authors are grateful to $\mathrm{Mr}$ Bultreys from FEI Europe Company for machining the notch with the help of a FEI dualbeam FIB/SEM system, to Dr Ian Sinclair from Southampton University for fruitful discussion on the $3 \mathrm{D}$ nature of the closure phenomenon and to Professor Lyndon Edwards from Open University for providing the fine grain Al material. The ID19 beam line
ESRF staff are acknowledged for the help and assistance during the tomographic imaging experiments.

This contribution is based on a presentation at the international workshop on 'Applications of X-ray tomography to study fracture and crack growth' held at Churchill College, Cambridge, UK in April 2005. The workshop was supported by Office of Naval Research Global under Department of the Navy Grant No. N00014-05-1-1043. The United States government retains a royalty free licence throughout the world in all copyrightable material contained herein.

\section{References}

1. J. C. Newman, Jr: Prog. Aerosp. Sci., 1998, 34, 347-390.

2. R. O. Ritchie and J. Lankford (eds.): 'Small fatigue cracks', 1-5; 1986, Warrendale, PA, The Metallurgical Society.

3. D. L. McDowell: Eng. Fract. Mech., 1996, 56, 357-377.

4. J. Husset, H. P. Lieurade, F. Maltrud and M. Truchon: Weld. World/Soudage Monde, 1985, 23, 276-283.

5. R. G. Forman and S. R. Mettu: Fract. Mech., 1992, 1, 519-546.

6. J. Baruchel: in 'X-ray tomography in material science', (ed. J. Baruchel et al.), 49-59; 2000, Paris, Hermes Science Publication.

7. W. Ludwig, J. Y. Buffière, S. Savelli and P. Cloetens: Acta Mater., 2003, 51, 585-598.

8. A. C. Kak and M. Slaney: 'Principles of computerized imaging'; 1988, New York, IEEE Press

9. J. C. Labiche, J. Segura-Puchades, D. Van Brussel and J. P. Moy: ESRF Newslett., 1996, 25, 41-43.

10. J. Y. Buffière, S. Savelli, P. H. Jouneau, E. Maire and R. Fougères: Mater. Sci. Eng. A, 2001, 316A, 115-126.

11. T. J. Marrow, J. Y. Buffière, P. J. Withers, G. Johnson and D. Engelberg: Int. Jal. Fatigue, 2004, 26, 717-725.

12. S. A. McDonald, M. Preuss, E. Maire, J. Y. Buffière, P. M. Mummery and P. J. Withers: Jal. Microscop., 2003, 209, 102-112.

13. R. Sinclair, M. Preuss, E. Maire, J.-Y.Buffière, P. Bowen and P. J. Withers: Acta Mater., 2004, 52, 1423-1438.

14. P. Cloetens, M. Pateyron-Salomé, J.Y. Buffière, G. Peix, J. Baruchel, F. Peyrin and M. Schlenker: Jal. Appl. Phys., 1997, 81, 5878-5886.

15. P. Cloetens, W. Ludwig, D. Van Dyck, J. Van Landuyt, J. P. Guigay and M. Schlenker: Appl. Phys. Lett., 1999, 75, 2912-2914.

16. E. Ferrié, J. Y. Buffière and W. Ludwig: Int. Jal. Fatigue, 2005, 27, 1215-1220.

17. A. Borbely, H. Mughrabi and G. Einsenmeier: Int. Jal. Fatigue, 2002, 115, 227-232.

18. Y. Murakami: 'Metal fatigue: defects of small defects and nonmetallic inclusions', Chap. 2; 2002, Oxford, Elsevier.

19. P. Clément, J. P. Angeli and A. Pineau: Fatigue Fract. Eng. Mater. Struct., 1984, 7, 251-265.

20. Y. Nadot, N. Ranganathan, J. Mendez and A. S. Béranger: $\underline{S c r}$. Mater., 1997, 37, 549-553.

21. K. S. Ravichandran: Fatigue Fract. Eng. Mater. Struct., 1997, 20 , 1423-1442.

22. J. Adrien: 'Optimisation des cycles thermiques appliqués aux fontes G. S. Ferritiques vis à vis des propriétés de fatigue', $\mathrm{PhD}$ thesis, INSA Lyon, Villeurbanne, France, 2004.

23. J. Y. Buffière, H. Proudhon, E. Ferrie, W. Ludwig and E. Maire: Nucl. Instr. Method. Phys. Res. B, 2005, 238B, 75-82.

24. E. Pereiro-Lopez, W. Ludwig and D. Bellet: Acta Mater., 2004, 52, 321-332.

25. E. Ferrié, J. Y. Buffière and W. Ludwig: Mater. Sci. Forum, 2005, 82, 227-230.

26. H. F. Poulsen: 'Three-dimensional X-ray diffraction microscopy', 205; 2004, Berlin, Springer.

27. E. Ferrie, J. Y. Buffière, W. Ludwig, A. Gravouil and L. Edwards: Acta Mater., 2006, 54, 1111-1122.

28. B. N. Cox and W. L. Morris: Eng. Fract. Mech., 1988, 31, 591-610.

29. K. T. Venkateswara Rao and R. O. Ritchie: Metall. Trans. A, 1991, 22A, 191-202.

30. J. Schijve: 'Fatigue of materials and structures', 174; 2001, Boston, MA, Kluwer Academics Publishers. 